\title{
A Control Method for SVG Based on Differential Geometry Nonlinear Control
}

\author{
Qi-Yong Pan ${ }^{1}$, Jin $\mathrm{Wu}^{2}$, Yi-Huai Wang ${ }^{2}$, Jing-Fei $\mathrm{Ni}^{2}$, Shan $\mathrm{Zhong}^{3}$ \\ ${ }^{1}$ College of Physics \& Electronic Engineering, Changshu Institute of Technology, Changshu, China \\ ${ }^{2}$ College of Computer Science \& Technology, Soochow University, Suzhou, China \\ ${ }^{3}$ Computer Science and Engineering College, Changshu Institute of Technology, Changshu, China \\ E-mail: panqiyong_1971@163.com \\ Received April 24, 2011; revised May 10, 2011; accepted May 17, 2011
}

\begin{abstract}
The control method for SVG is researched in this paper. Based on the working mechanism of SVG, the logic switch function is introduced to establish the dynamic mathematic model. A differential geometry variable control method is provided and the differential geometry linear theory is used to convert the nonlinear system to a linear system. Then based on the former work the control of SVG is devised. Finally, the control of SVG is simulated and the result shows the differential geometry nonlinear control is robust and stable comparative to the traditional PID control method and it is an effective to control the SVG.
\end{abstract}

Keywords: Mathematic Model, Nonlinear, Differential Geometry, Control

\section{Introduction}

Static Var Generator (SVG) as one of the flexible AC transmission systems (FACTS) devices can dynamically compensate the reactive power, and the characters of the smooth reactive power adjusting and the rapid dynamics performance makes it obtained the widely attention from inside and outside of the country [1-3].

The devising of the control model for SVG is the key problem of SVG. In the respective works, the literature [4] established the single nonlinear equivalent circuit model, [5] built the dynamic model of SVG based on the three phase and using the ratio coefficient $\mathrm{K}$ to describe the relations of network side voltage and the direct capacity voltage, without the considering of working mechanism and the dynamical behaviors.

With the development of the modern control theory, the control method is widely used such as the advanced PID control, the nonlinear robust control, predicting fuzzy control, inverse control and the neutral control [6]. And the above control parameters are generally using the experience, but some parameters of SVG is unpredictable, and sometimes is changed according with the time, so the optimum control parameters is hard to be obtained and the system control quality is also difficult to be satisfied.

In order to make the system have the relative perfect compensation performance and the relative high control precise. Through the dynamics of the SVG, the dynamic mathematics of SVG is established, and based on the dynamic mathematics model, the control method based on differential geometry structure and using the differential geometry precise linear theory, the nonlinear system is converted to the linear system, and then the control is devised by applying the nonlinear variable structure control theory exponent tending law. Finally, the control system is analyzed by using the differential geometry control method and improving the control precise.

\section{The Dynamic Mathematics of SVG}

The main circuit structure of SVG is showed as Figure 1, and IGCT [7] is used as the main switch tube of voltage inverter.

On suppose of the research precise is not affected, the below condition is assumed:

1) Power switch device is the ideal switch;

2) Grid voltage is the three phase cosine voltage;

3) All the losses of devices including the loss of converter itself and the loss of transformer are denoted using equivalent resistance $\mathrm{R}$;

4) The leakage of transformer and the connecting reactance is using the equivalent inductance $\mathrm{L}$ to represent;

5) Ignore the voltage harmonics wave weight of invert AC side. 


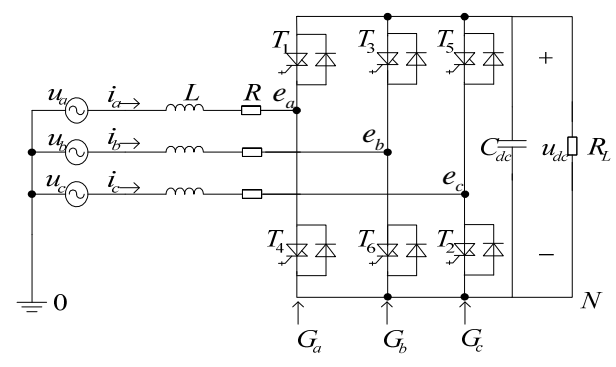

Figure 1. Circuit configuration of SVG.

The three-phase voltage is as Equation (1):

$$
u_{a b c}=\left[\begin{array}{l}
u_{a} \\
u_{b} \\
u_{c}
\end{array}\right]=U_{m}\left[\begin{array}{c}
\cos \omega t \\
\cos \left(\omega t-\frac{2}{3} \pi\right) \\
\cos \left(\omega t+\frac{2}{3} \pi\right)
\end{array}\right]
$$

In the Equation (1), $U_{m}$ is the max value of system linear voltage. (2):

The voltage of inverter $\mathrm{AC}$ side is showed as Equation

$$
e_{a b c}=\left[\begin{array}{c}
e_{a} \\
e_{b} \\
e_{c}
\end{array}\right]=\sqrt{\frac{2}{3}} \cdot K \cdot u_{d c}\left[\begin{array}{c}
\cos (\omega t+\delta) \\
\cos \left(\omega t-\frac{2}{3} \pi+\delta\right) \\
\cos \left(\omega t+\frac{2}{3} \pi+\delta\right)
\end{array}\right]
$$

In Equation (2), where $K$ is the ratio of inverter output linear voltage with the direct current side voltage, and $\delta$ is the phrase of device voltage and the system voltage.

In order to better analyze, the logic switch function $G_{i}$ is introduced, the states of three bridge arm is using $G_{a}, G_{b}$ and $G_{c}$ to represent, when the condition are turning on the above bridge arm and turning off the below bridge arm, the $G_{a}, G_{b}$ and $G_{c}$ are 1, and while the condition are turning off the above bridge arm and turning on the below bridge arm, the $G_{a}, G_{b}$ and $G_{c}$ are 0 .

According to the Kirchhoff's voltage law, the a-phase loop equation is as Equation (3):

$$
L \frac{\mathrm{d} i_{a}}{\mathrm{~d} t}+R i_{a}+\left(u_{a N}+u_{N 0}\right)=u_{a}
$$

When $G_{a}=1$ then $u_{a N}=u_{N 0}$; while $G_{a}=0$ then $u_{a N}=0$. So Equation (2) can be written as bellow:

$$
L \frac{\mathrm{d} i_{a}}{\mathrm{~d} t}+R i_{a}+\left(u_{d c} G_{a}+u_{N 0}\right)=u_{a}
$$

The $b$ and $c$ phase equation also can be obtained, as for the three-phase input, there is no midline, so $i_{a}+i_{b}+i_{c}=0$, If the three phase power voltage is in balance, then

$$
u_{N 0}=-\frac{1}{3}\left(G_{a}+G_{b}+G_{c}\right) u_{d c}
$$

The inverter is comprised of six power switch tubes showed in Figure 1, and the on-off rule is as the same bridge arm can be on at the same time.

Combined with the above switch function, the three bridge arm just has two states as " 1 " and " 0 ".

$T_{1}, T_{3}$ and $T_{5}$ forms eight models such as 000, 010, $011,100,101,110$ and 111. The switch model of 000 and 111 make the output voltage of invert as 0 , so the two switch models is at the state 0 .

The Kirchhoff current rule is applied in direct current side capacitance positive node as Equation (6)

$$
C_{d c} \frac{\mathrm{d} u_{d c}}{\mathrm{~d} t}=i_{a} G_{a}+i_{b} G_{b}+i_{c} G_{c}-\frac{u_{d c}}{R_{L}}
$$

For the circuit equation, it is very complex at the coordinate at $a, b$ and $c$, the d-q transform is used in the equation of the three-phase voltage current, and the transform matrix is showed as below:

$$
C=\sqrt{\frac{2}{3}}\left[\begin{array}{ccc}
\cos \omega t & \cos \left(\omega t-\frac{2}{3} \pi\right) & \cos \left(\omega t+\frac{2}{3} \pi\right) \\
-\sin \omega t & -\sin \left(\omega t-\frac{2}{3} \pi\right) & -\sin \left(\omega t+\frac{2}{3} \pi\right) \\
\frac{\sqrt{2}}{2} & \frac{\sqrt{2}}{2} & \frac{\sqrt{2}}{2}
\end{array}\right]
$$

Then the mathematics model of SVG in the coordinate $\mathrm{d}-\mathrm{q}$ is showed as Equation (8):

$$
\begin{aligned}
\frac{\mathrm{d}}{\mathrm{d} t}\left[\begin{array}{c}
i_{d} \\
i_{q} \\
u_{d c}
\end{array}\right] & =\left[\begin{array}{ccc}
-\frac{R}{L} & \omega & -\frac{K}{L} \cos \delta \\
-\omega & -\frac{R}{L} & -\frac{K}{L} \sin \delta \\
\frac{K}{C_{d c}} \cos \delta & \frac{K}{C_{d c}} \sin \delta & 0
\end{array}\right] \\
& \times\left[\begin{array}{c}
i_{d} \\
i_{q} \\
u_{d c}
\end{array}\right]+\frac{1}{L}\left[\begin{array}{c}
U_{m} \\
0 \\
0
\end{array}\right]
\end{aligned}
$$

\section{The Differential Geometry Nonlinear Control of SVG}

\subsection{The Control Theory of Differential Geometry for Nonlinear System}

For the given multi-variable nonlinear system: 


$$
\left\{\begin{array}{l}
\dot{X}=f(X)+\sum_{i=1}^{m} g_{i}(X) \cdot u_{i} \\
y_{j}=h_{j}(X), j=1, \cdots, m
\end{array}\right.
$$

In the Equation (9), $X$ is the local coordinate defined on the $n$-dimension $C^{\infty}$ fluent $M$, and $f, g_{1}, \cdots, g_{m}$ are the vector field; $h$ is the mapping of $C^{\infty}$ in $M$ as $h: M \rightarrow R^{j}$, and $y$ is the output.

If $r_{i}$ is the max positive integer satisfied the below conditions, then

$$
\left\{\begin{array}{l}
{\left[L_{g 1} L_{f}^{r_{i}-1} h_{i}(X), \cdots, L_{g m} L_{f}^{r_{i}-1} h_{i}(X)\right] \neq 0, X \in M} \\
{\left[L_{g 1} L_{f}^{k} h_{i}(X), \cdots, L_{g m} L_{f}^{k_{i}} h_{i}(X)\right]=0, k_{i}<r_{i}-1, X \in M}
\end{array}\right.
$$

In the Equation (10), $L_{f}^{r_{i}-1} h_{i}(X)$ is the $r_{i}-1$ order inverse derivative of $h_{i}(X)$ to $f(X)$ while $L_{g m} L_{f}^{r_{i}-1} h_{i}(X)$ is the inverse derivative of $L_{f}^{r_{i}-1} h_{i}(X)$ to vector field $g(X)$, where $D(X)$ and $E(X)$ are showed as follows:

$$
\begin{gathered}
D(X)=\left[\begin{array}{c}
L_{g 1} L_{f}^{r_{1}-1} h_{1}(X), \cdots, L_{g m} L_{1}^{r_{m}-1} h_{1}(X) \\
\cdots \\
L_{g 1} L_{f}^{r_{1}-1} h_{m}(X), \cdots, L_{g m} L_{1}^{r_{m}-1} h_{m}(X)
\end{array}\right] \\
E(X)=\left[\begin{array}{c}
L_{f}^{r_{1}} h_{1}(X) \\
\cdots \\
\cdots \\
L_{f}^{r_{m}} h_{m}(X)
\end{array}\right]
\end{gathered}
$$

Definition 1. The sufficient and necessary condition for the decoupling of nonlinear system is the matrix $D(X)$ is non-singular, and the feedback control rule is as follows:

$$
u=\alpha(X)+\beta(X) v=-[D(X)]^{-1} E(X)+[D(X)]^{-1} v
$$

So the system is decoupled on $M$ showed as Equation (12)

$$
\left\{\begin{array}{l}
\dot{X}=f(X)+g(X) \alpha(x)+g(X) \beta(X) v \\
y_{j}=h_{j}(X),(j=1,2, \cdots, m)
\end{array}\right.
$$

\subsection{The Entire Linearizing of SVG}

According to the Equation (8), the dynamic model of $\mathrm{SVG}$ is the non-linear couple relation. Therefore, the decoupling should be satisfied at first.

The state variable is defined as $x_{1}=i_{d}, x_{2}=i_{q}$ and $x_{3}=u_{d c}$, using $u=(\sin \delta \cos \delta)^{\mathrm{T}}$ to control the input, at the same time using $y_{1}=i_{d}$ and $y_{2}=i_{q}$ as the output. Therefore, the non-linear model of SVG can be rep- resented as the affine non-linear forms:

$$
\left\{\begin{array}{l}
\dot{X}=f(X)+\sum_{i=1}^{2} g_{i}(X) \cdot u_{i} \\
y_{1}=h_{1}(X) \\
y_{2}=h_{2}(X)
\end{array}\right.
$$

In the Equation (13), where

$$
f(X)=\left[\begin{array}{c}
-\frac{R}{L} x_{1}+\omega x_{2} \\
-\omega x_{1}-\frac{R}{L} x_{2} \\
0
\end{array}\right] g(X)=\left[\begin{array}{cc}
0 & -\frac{K}{L} x_{3} \\
\frac{K}{L} x_{3} & 0 \\
\frac{K}{C_{d c}} x_{3} & \frac{K}{C_{d c}} x_{3}
\end{array}\right]
$$

The affine non-linear system such as Equation (9), according to the Equation (10), the below equations can be obtained:

$$
\begin{gathered}
L_{g_{1}} h_{1}(X)=0 ; \\
L_{g_{2}} h_{1}(X)=0 ; \\
L_{f} h_{1}(X)=-\frac{R}{L} x_{1}+\omega x_{2} ; \\
L_{g_{1}} L_{f} h_{1}(X)=\frac{K}{L} x_{3} ; \\
L_{g_{2}} L_{f} h_{1}(X)=-\frac{K}{C_{d c}} x_{3} ; \\
L_{g_{2}} h_{1}(X)=0 ; \\
L_{g_{2}} h_{2}(X)=0 ; \\
L_{f} h_{2}(X)=-\omega x_{1}+\frac{R}{L} x_{2} ; \\
L_{g_{1}} L_{f} h_{2}(X)=-\frac{K}{L} x_{3} ; \\
L_{g_{2}} L_{f} h_{2}(X)=-\frac{K}{C_{d c}} x_{3} ;
\end{gathered}
$$

From the above equations we can know the matrix $D(X)$ is not singular and satisfying the conditions for decoupling of input and output.

The state equations on the new coordinate are as bellows:

$$
\left\{\begin{array}{l}
\dot{z}_{1}^{1}=z_{1}^{2} \\
\dot{z}_{1}^{2}=L_{f}^{2} h_{1}(X)+L_{g} L_{f} h_{1}(X) u^{\prime} \\
\dot{z}_{2}^{1}=z_{2}^{2} \\
\dot{z}_{2}^{2}=L_{f}^{2} h_{2}(X)+L_{g} L_{f} h_{2}(X) u^{\prime}
\end{array}\right.
$$


In Equation (14), $u^{\prime}=[\sin \delta \cdot \cos \delta]^{\mathrm{T}}$, and the system represented by Equation (14) can be divided to two independent subsystems.

$$
\left\{\begin{array}{l}
{\left[\begin{array}{l}
\dot{z}_{1}^{1} \\
\dot{z}_{1}^{2}
\end{array}\right]=\left[\begin{array}{ll}
0 & 1 \\
0 & 0
\end{array}\right]\left[\begin{array}{l}
z_{1}^{1} \\
z_{1}^{2}
\end{array}\right]+\left[\begin{array}{l}
0 \\
1
\end{array}\right] v_{1}} \\
{\left[\begin{array}{l}
\dot{z}_{2}^{1} \\
\dot{z}_{2}^{2}
\end{array}\right]=\left[\begin{array}{ll}
0 & 1 \\
0 & 0
\end{array}\right]\left[\begin{array}{l}
z_{2}^{1} \\
z_{2}^{2}
\end{array}\right]+\left[\begin{array}{l}
0 \\
1
\end{array}\right] v_{2}}
\end{array}\right.
$$

In Equation (15), $v_{1}$ and $v_{2}$ are the virtual input value, according the Equation (15), the factual input control rule of the system is as Equation (16)

$$
\begin{gathered}
{\left[\begin{array}{c}
i_{d} \\
i_{q} \\
u_{d c}
\end{array}\right]=D^{-1}(X)\left[\begin{array}{l}
v_{1}-E_{1}(X) \\
v_{2}-E_{2}(X) \\
v_{3}-E_{3}(X)
\end{array}\right]} \\
E(X)=\left[\begin{array}{l}
E_{1}(X) \\
E_{2}(X) \\
E_{3}(X)
\end{array}\right]=\left[\begin{array}{l}
L_{f}^{2} h_{1}(X) \\
L_{f}^{2} h_{2}(X) \\
L_{f} h_{3}(X)
\end{array}\right]
\end{gathered}
$$

Therefore, the system of SVG is linearized as the decoupled system.

\section{The Simulation and Analysis}

In order to verify the advantage of the statistics of the
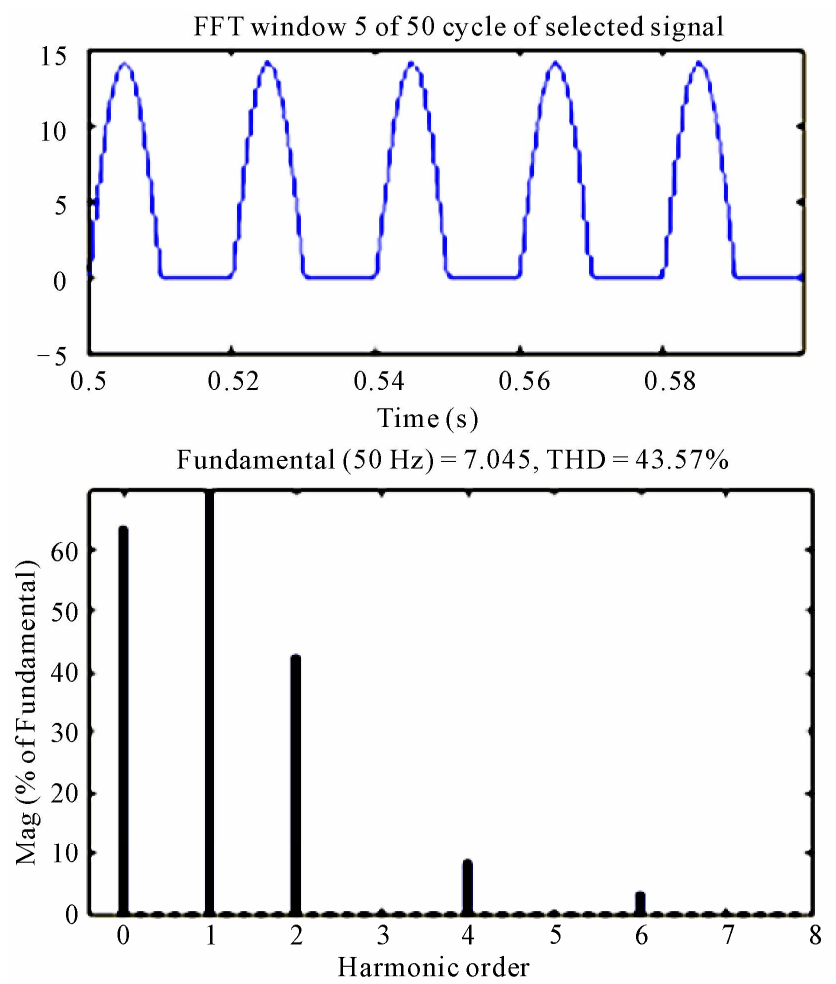

system, the differential geometry structure control and the traditional PID control method on the same conditions are simulated by using the software Matlab to simulate.

The simulation conditions are as follows: the voltage of the power supply is $380 \mathrm{~V}$, the system frequency is 50 $\mathrm{Hz}$, the connecting inductance of device to system is 0.01 $\mathrm{H}$, and the side capacitance of device direct is $0.003 \mathrm{~F}$, the equivalent resistance is $22 \Omega$.

The current voltage and FFT before and after compensation are showed respectively as Figure 2 and Figure 3.

From the figure, we can found the grid current and the FFT is showed in Figure 3 before the compensation is $43.57 \%$ and after the compensation by the differential geometry structure, the content of THD is reduced effectively to $10.84 \%$. The aberrance of the grid voltage is improved largely. The method proposed in this paper is proved as a feasible method having good performance.

\section{Conclusions}

From the dynamic performance of working mechanism of SVG, the dynamic mathematics of SVG is established by introducing the logic switch functions. The control is simplified to affine non-linear form by using the differential geometry knowledge, and then through the differ-

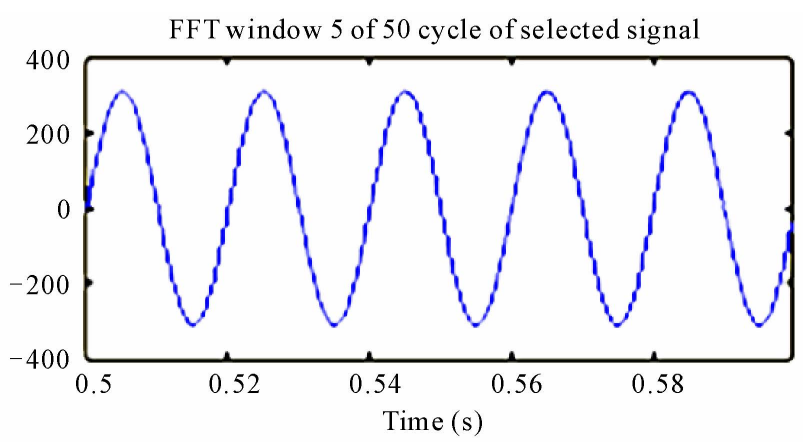

$\times 10^{-14}$ Fundamental $(50 \mathrm{~Hz})=311, \mathrm{THD}=0.00 \%$

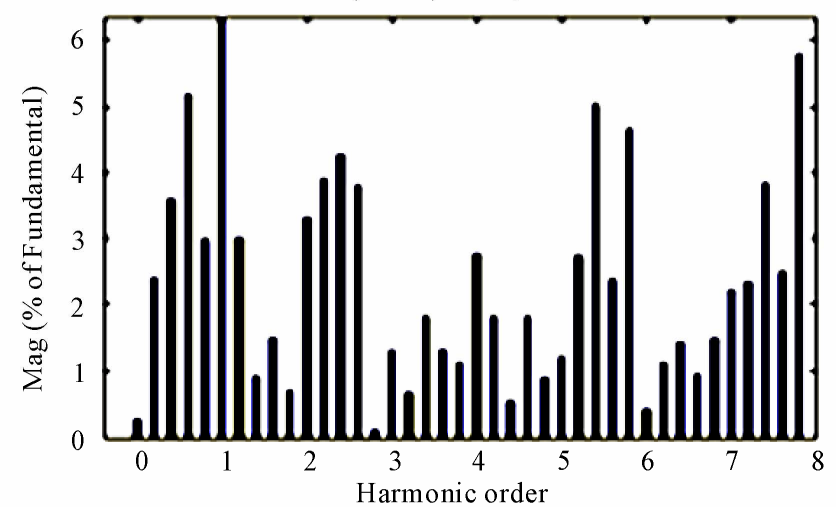

Figure 2. Simulation waveforms before compensation. 

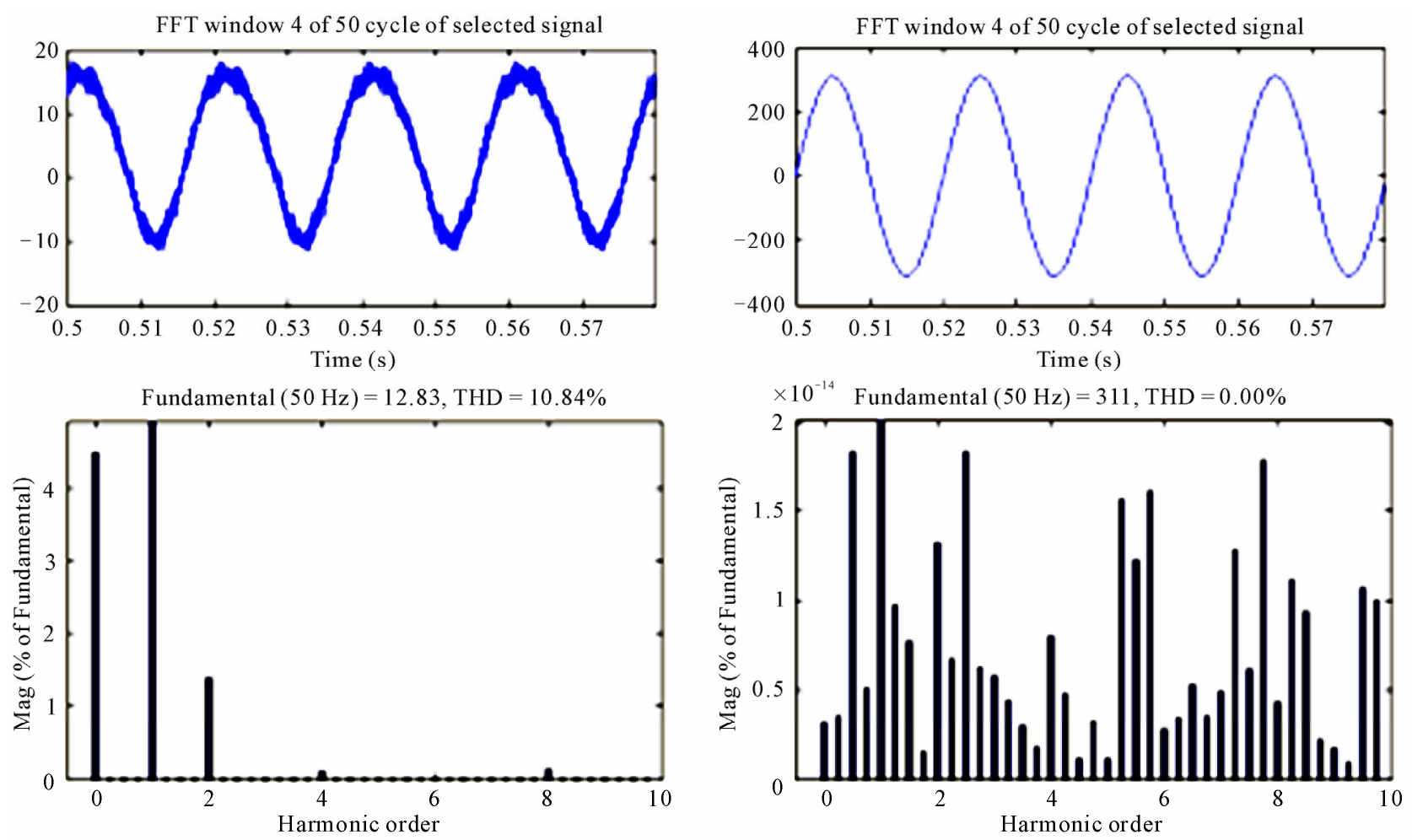

Figure 3. Simulation waveforms after compensation.

ential geometry transform to realize its non-linear decoupling control, which makes the non-linear control problem to the simple linear control problem and also avoid the parameters of the SVG system absorbing and taking the negative effect to the control. Then the differential geometry control is devised. Finally, the experiment is simulated which shows the differential variable structure is more robust ${ }^{[8]}$ and stable than the traditional PID control.

\section{References}

[1] R. Adapa, "FACTS System Studies," IEEE Power Engineering Review, Vol. 22, No. 12, 2002, pp. 17-22. doi:10.1109/MPER.2002.1098039

[2] Y. Z. Sun and Q. J. Liu, "A Summary of Facts Control Technology-Model, Objective and Strategy," Automation of Electric Power Systems, Vol. 23, No. 6, 1999, pp. $1-5$.

[3] Q. R. Jiang, X. R. Xie and J. Y. Chen, "Shunt Compensa- tion of Power System - Structure, Fundametal, Control and Application,” Industry Press, Beijing, 2004.

[4] Z. A. Wang, J. Yang, J. J. Liu, et al., "Harmonic Suppression and Reactive Power Compensation," Industry Press, Beijing, 2005.

[5] X. Tang, A. Luo and C. M. Tu, "A New Method to Current Control of Active Power Filter," Automation of Electric Power Sytems, Vol. 28, No. 13, 2004, pp. 31-34.

[6] W. Chen and J. Wu, "Adaptive Control of ASVG by Using Diagonal Recurrent Neural Network," Automation of Electric Power Systems, Vol. 23, No. 9, 1999, pp. 33-37.

[7] G. C. Cho, G. H. Jung, N. S. Choi and G. H. Cho, "Analysis and Controller Design of Static Var Compensator Using Three-Level GTO Inverter," IEEE Transactions on Power Electronics, Vol. 11, No. 1, 1996, pp. 57-65.

[8] S. K. Nguang, "Robust Stabilization for A Class of Time-Delay Nonlinear Systems," IEEE Transactions on Automatic Control, Vol. 45, No. 4, 1994, pp. 756-762. doi:10.1109/9.847117 